\title{
Preparation and use of fixative slides for the diagnosis of Trichomonas vaginalis
}

\author{
C. R. AMIES AND MARIA GARABEDIAN \\ From Ontario Department of Health, Central Laboratory, Toronto, Canada
}

SYNOPSIS Clean microscopic slides are dipped in a solution of mercuric chloride and sucrose in $30 \%$ alcohol and allowed to dry. A thin transparent film of cytological fixative is thus formed on both sides of the slide. Fixative slides, prepared in this manner, can be stored indefinitely provided that they are not exposed to a warm, humid atmosphere. When a drop of fluid containing bacteria, protozoa, or mammalian cells is spread over the surface of such a slide the formed elements are immediately fixed, the finer cytological details being well preserved. Staining and examination are carried out when the specimen arrives at the laboratory. This method is particularly suitable for a diagnostic laboratory service in which most of the specimens arrive by mail.

The method has been successfully employed in the diagnosis of Trichomonas vaginalis infection of the female genital tract. This parasite was found by phase-contrast microscopy in $47 \%$ of 1,000 women examined at a police court clinic. Fixative slide preparations were also obtained from these women at the time of their medical examination. Of these specimens, $38.6 \%$ showed the presence of $\mathrm{Tr}$. vaginalis, and $10 \%$ were also found to be infected with $N$. gonorrhoeae.

The fixative slide technique may also prove of value in such widely separated fields as exfoliative cytology and the diagnosis of primary syphilis.

For satisfactory results in pathological investigations it is essential that the material to be studied should be either in a fresh condition at the time of examination or adequately fixed immediately after removal from the body. This is of the utmost importance in the diagnosis of malignant disease by examination of exfoliated cells, and similar requirements must be satisfied in the case of delicate protozoa and other organisms which quickly disintegrate when removed from their normal environment. When smears from tissues or exudates have to be studied the usual procedure up to now has been to spread the material thinly on a microscope slide and to fix the film so prepared in alcohol or other solution before it has time to dry. Often, however, treatment of the specimen is delayed, the film dries, and the cells are damaged. Equally unsatisfactory is the sending of dry unfixed smears to the laboratory for diagnosis of Trichomonas vaginalis infection. In order to overcome these difficulties we have devised a method which allows fixation to occur immediately the cells are brought into contact with the surface of a chemically treated microscope slide. Most of our experience so far has been gained with Trichomonas vaginalis infection of the female genital tract but the results have been so encouraging that it seems justifiable to describe the methods without waiting for the findings of similar investigations in other fields.

\section{EXPERIMENTAL}

The idea of coating a microscope slide with a dry film of fixative proved to be more difficult in practice than was at first anticipated. The choice was limited to a few of those fixatives currently used in cytology because most contain volatile substances. It was essential that the fixative solution when applied to the glass surface and allowed to dry should produce a film which was stable under normal storage conditions, yet this film must dissolve instantly in a few drops of fluid spread over the slide. It was also necessary to counteract the shrinking effect of the main fixative by adding a substance capable of producing a swelling of cell protoplasm. Acetic acid usually fulfils this function. This, being volatile, was inadmissible, but sodium acetate proved to be an excellent substitute. In addition to preventing shrinkage this salt also neutralizes the excessive acidity of the fixatives usually employed and thus maintains the $p \mathrm{H}$ at any desired level. Attention had also to be given to the effects of salt concentration, particularly in the case of the more 
delicate protozoa which are even more vulnerable than mammalian cells. Another desirable though not essential condition was that the film of fixative should be transparent, for it seemed likely that an opaque film would be less acceptable to our colleagues. To these problems were added some unforeseen difficulties concerning the chemical cleanliness of the glass microscope slides. These were of the pre-cleaned type, that is, they had received treatment at the factory in order to render the surface wettable. The process appears to be a trade secret. Imperfectly cleaned slides could not be coated with a uniform film of any of the aqueous fixatives tested. This difficulty was finally overcome by using an alcoholic solvent.

\section{FORMULATION OF A SUITABLE FIXATIVE}

The earliest experiments were carried out with microscope slides which had been dipped in Zenker's bichromatemercury perchloride solution and allowed to dry in a vertical position. Fairly good fixation of $T r$. vaginalis was obtained but the unsightly appearance of the crystalline deposit on the slide was a serious disadvantage. After many experiments it was discovered that this crystallization could be prevented and fixation be improved by adding a carbohydrate to the solution. The addition of $5 \mathrm{~g}$. of lactose and $1 \mathrm{~g}$. of sodium acetate to $100 \mathrm{ml}$. of Zenker's fluid produced a solution which yielded a transparent yellow film free from residual stickiness. This solution, however, is unstable, the $\mathrm{HgCl}_{2}$ being rapidly converted to the insoluble compound $\mathrm{HgCl}$ (calomel) by the reducing sugar lactose. For this reason it was necessary to complete the preparation of the slides within one hour of mixing the fixative. A further disadvantage of this formula was the gradual change in colour of the film from yellow to brown after exposure to the air for several weeks, but this did not occur when the slides were packed in a box and stored in a dry place. The modified Zenker formula was given an extensive trial. It fixed well $T r$. vaginalis and the cells composing the inflammatory exudate and would have been adopted if the disadvantages above noted could have been overcome. However, as complete stability was regarded as an essential requirement, this formula was abandoned and further work was undertaken to find a more satisfactory one.

Experiments were next carried out with films containing $\mathrm{HgCl}_{2}$ as the sole fixing agent. There was a good precedent for this. According to Lillie (1954), Giemsa himself recommended Schaudinn's 'sublimate alcohol' for wet film fixation and this method still finds favour among protozoologists. Now that potassium bichromate had been eliminated from the formula it was possible to employ sucrose, a non-reducing substance, to maintain transparency of the dry film. Although this sugar is only slightly soluble in absolute alcohol it will readily dissolve in $30 \%$ alcohol, and from this solution a transparent uniform film can be deposited on a clean glass surface. The complete formula is as follows:

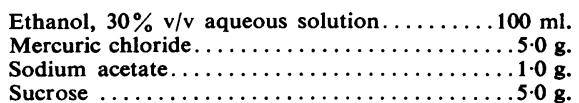

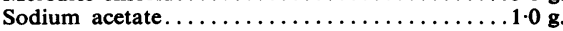

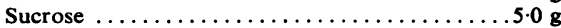
Chemicals of reagent quality should be used and the
solution must be filtered through hard filter paper. It has: a $p \mathrm{H}$ of $5 \cdot 7$. Films prepared from this fixative remain unchanged unless they are exposed to a warm and humiff atmosphere. Under the latter conditions the slides be $\frac{f}{0}$ come slightly cloudy but this appears to be a purely physical change which does not affect their fixative properties. The slides could, therefore, be used in some tropical climates but under very humid conditions it may be advisable to use lactose instead of sucrose in theformula given above. In this case, the slides should be prepared within two hours after mixing the solution.

\section{TECHNIQUE OF MAKING FIXATIVE SLIDES}

The fixative solution should be prepared freshly and itpo should be kept in a scrupulously clean glass vessel such as a coplin jar fitted with a screw cap. As a safeguard against dermatitis the operator should wear rubbero gloves and should be cautioned against allowing the mercury-containing solution to come into contact with the eyes or skin. The work should be carried out in क dust-free room with a dry atmosphere. Microscope slides with an etched surface at one end are recommended Each slide is removed in turn from the box and dippedmomentarily in the fixative. It is then drained againsto the side of the glass jar and set to dry in an aluminium $r$ rack specially designed to hold 50 slides in such a position that the metal does not come into contact with the wet fixative. A strip of filter paper on which the slides rese removes excess fixative which has drained to the lowero edge. Drying at $37^{\circ} \mathrm{C}$. for a short time is permissible buf the slides should not be held for more than two hours a this temperature. Overnight drying at room temperature is preferred. After examination to ascertain that they are no longer sticky the slides are packed in their originab boxes and stored in a cool, dry place. They should remain in good condition indefinitely. The boxes and slides must be appropriately labelled so that the latter aref not confused with ordinary untreated slides. This is necessary because fixative-treated slides cannot be em ployed for some routine purposes such as the making of blood films: the plasma proteins are coagulated so rapidly that there is insufficient time for making a blood film.

SOME PROPERTIES OF THE FIXATIVE FILM

It is outside the scope of this article to discuss the physican nature of the film of fixative but a few easily ascertainable facts will contribute to an understanding of how fixation is brought about.

The amount of $\mathrm{HgCl}_{2}$ deposited on unit surface of the microscope slide was easily determined by dissolving the dry film in a known volume of water and submitting the solution to chemical analysis. It was found that the amount deposited was $0.04 \mathrm{mg} . / \mathrm{cm}^{2}$ of glass surface? The mean thickness before drying takes place was cal culated to be $0.8 \mu$; after drying the thickness is less than a wavelength of light because the coated surface of the slide shows the diffraction effect.

The presence of the inorganic salts in the film can be visually demonstrated very simply. The fixative solution 
is prepared without the addition of carbohydrate. Slides dipped into this solution and allowed to dry in the usual manner show a heterogeneous crystalline deposit. On the other hand, microscopic examination (phase contrast) of a film prepared from the normal fixative shows either complete transparency or the beginning of very fine crystallization at scattered points where dust particles or other impurities have acted as 'seeds'. Films sometimes develop a bluish opalescence after long storage: this is of no consequence.

One drop $(0.05 \mathrm{ml}$.) of inflammatory exudate or other pathological fluid when spread over the surface of a prepared slide will dissolve the film and form approximately a $1 \%$ solution of the $\mathrm{HgCl}_{2}$. It was important to know how quickly solution takes place because on this factor depends the effectiveness of the technique. This was studied by using slides which had been partly immersed in the fixative so that the film extended over only one-half of the glass surface. A cover glass was placed over the dry slide and the margin of the film of fixative was focused with the phase-contrast microscope. A drop of fluid containing living $T r$. vaginalis was then allowed to run under the cover glass by capillary action. When the advancing fluid reached the edge of the film of fixative the protozoa instantly became immobile, the flagella remained intact, and the nucleus became clearly visible. The fixation of mammalian cells was studied in a similar manner except that in this case the slide half covered with a film of dry fixative was observed under the dark-field microscope. A washed suspension of leucocytes prepared from fresh blood was introduced as before between slide and cover glass. Viability of the polymorph cells was indicated by the ceaseless motion of the intracytoplasmic granules. When these cells came into contact with the film of fixative all movement of the granules abruptly ceased and the nucleus became more prominent. There was very little alteration in the size of the cell when fixation took place.

It was also important to establish the fact that cells brought into contact with the fixative film become firmly adherent to the glass surface and that they are not washed off during the subsequent stages of washing and staining. To prove this, about $1.0 \mathrm{ml}$. of water was placed on a fixative slide bearing a film of vaginal exudate. After a few minutes this wash fluid was collected in a test tube. The washings from 20 such smear preparations were pooled and centrifuged, and the deposit so obtained was examined microscopically for the presence of formed elements. Only a few fragments of keratin were seen. Direct confirmation of this finding was obtained by microscopic examination of marked areas of the same smear preparation before and after treatment with water and Gram stain solutions. No loss of cells was revealed.

\section{RESULTS OBTAINED IN TRICHOMONAS VAGINALIS INFECTIONS}

There is no doubt that the most reliable method of detecting $T r$. vaginalis is the examination of wet mounts, preferably with the phase-contrast microscope. The characteristic motility of this organism makes its recognition easy, even when it is present only in small numbers. This method was our basis for comparison in evaluating the fixative film technique. Material for study was obtained from a clinic attached to a police court where women indicted for prostitution receive medical examination and treatment. Swabs of the cervix and urethra taken by the medical officer and the nursing staff were forwarded to the laboratory in transport medium (Stuart, Toshach, and Patsula, 1954), together with smears prepared on fixative slides. All swabs were examined for $T r$. vaginalis as described above and then cultured in Peizer medium (Trowbridge and McConkey, 1944) for evidence of gonococcal infection. This was done within a few hours of the time of collecting the specimen. The smear preparations were not processed until the following day. These were arranged in racks and immersed in a stream of gently running water for two to five minutes. They were then stained by Gram's method, using a standard procedure with saffranin as counterstain. The Gram's iodine solution effectively removed any deposit of mercury salts. An oil immersion objective was used for examination in order to recognize both $N$. gonorrhoeae and $T r$. vaginalis. The latter, if present, were usually easily recognized by the presence of the flagella. Only in the case of effete organisms were these structures displaced and no longer visible. In addition, both the nucleus and the cytoplasm of this protozoon have characteristics which aid in their recognition.

The results obtained from the examination of 1,000 specimens submitted by the police court clinic are given in Table $\mathrm{I}$.

\section{TABLE I}

RESULTS IN 1,000 SPECIMENS FROM A POLICE COURT CLINIC

Number showing $T r$. vaginalis

In wet mounts examined by phase microscopy

In smears prepared on fixative slides

Success of fixative slide technique expressed as a percentage of results obtained with wet mounts

Number showing $N$. gonorrhoeae

$$
\begin{array}{r}
471(47.1 \%) \\
386(38.6 \%) \\
81.9 \% \\
107(10.7 \%)
\end{array}
$$

Further analysis of the results showed that failure to demonstrate $T r$. vaginalis in the fixed smears usually occurred in cases in which the wet mount results were recorded as showing few organisms. Light infections of this kind are probably of little significance. In this series there were 24 instances of a positive finding in the stained film with a negative result in the corresponding wet mount preparation.

During the course of these studies it was noticed that spirochaetes resembling Borrelia vincenti, often found in vaginal discharges, are well preserved by the fixative slide technique. This suggested that the method might be of use as a means of demonstrating 
Treponema pallidum in dry smears of fluid obtained from primary chancres. Using fixative slides, our colleague Mr. H. B. Smith, head of the Division of Serology, kindly prepared a number of smears of rabbit testis suspension containing Reiter spirochaetes. These were stored for several weeks. A drop of water was then placed on each smear, a cover glass was placed on top and the preparations were examined by dark-field microscopy. The spirochaetes had retained their morphology and were easily recognized. This technique deserves to be properly evaluated at a venereal diseases centre where clinical material is available.

\section{COMMENT}

At this laboratory about 3,000 smears of cervical exudate are examined each month. These are in addition to those received from the police court clinic: they are sent by hospitals and private practitioners, and many are accompanied by a swab specimen in transport medium for culture. These swabs are not examined for $T r$. vaginalis because delay in transit, often as long as $\mathbf{4 8}$ hours, makes this an unreliable procedure. The Ontario Health Department issues free mailing boxes to doctors and hospitals on request. Each box contains two microscope slides, a specimen bottle containing transport medium, and a sterile cotton-tipped applicator for use as a swab. When fixative slides are issued instead of plain slides it will be possible to report on the presence or absence of $T r$. vaginalis. ${ }^{1}$ At present the stained smears only provide information about the nature of the cells in the exudate and about the finding of Gram-negative diplococci.

${ }^{1}$ The fixative film method was used as a routine throughout the year 1963. Of 12,455 specimens submitted for examination $1,986(15.9 \%)$ showed the presence of $\mathrm{Tr}$. vaginalis.
Up to now, we have prepared our own fixative slides but this has become a routine procedure which: could be undertaken by any conscientious laborator $\vec{P}$ attendant. About 150 fixative slides can be prepare $\Phi$ in one hour. Once this method has gained acceptance the slides could be made on a commercial scale at reasonable cost. This would probably result in improvement in the uniformity of the film by adopting a mechanized process.

Fixation slides may find their most important use in the field of diagnostic cytology, to some extent replacing wet fixation with alcohol and othe? volatile mixtures. This applies particularly te situations where the specimen must be transported to the laboratory by mail. Preliminary studies have satisfied us that malignant cells in sputum would ba well enough preserved to allow their recognitiob without difficulty. We do not claim that fixative slides always give results which equal the wet fixation methods but the advantages of the formes entitle them to serious consideration. Further wor on these lines is in progress.

We are grateful to Mr. Charles Rhodes of the Industria Hygiene Laboratory for undertaking a number of mercury determinations; to Mr. Ralph S. Bull, M.A., and Mro R. G. Reynolds, B.Sc., of the Biochemistry Division we extend our thanks for useful advice on chemical matters The cooperation of the medical and nursing staff of the police court clinic is gratefully acknowledged.

\section{REFERENCES}

Lillie, R. D. (1954). Histopathologic Technic and Practical Hist chemistry, p.38. Blakiston, New York.

Stuart, R. D., Toshach, S. R., and Patsula, T. M. (1954). Canad. $\bar{D}$ publ. Hlth, 45, 73.

Trowbridge, M. Jr., and McConkey, R. M. (1944). War Med. (Chic. 5,36 . 